\title{
Correction to: How Does the Coupling of Real-World Policies with Optimization Models Expand the Practicality of Solutions in Reservoir Operation Problems?
}

\section{Gi Joo Kim ${ }^{1}$ (D) Young-Oh Kim ${ }^{1}$}

Published online: 23 September 2021

(c) Springer Nature B.V. 2021

\section{Correction to: Water Resour Manag (2021) 35:3121 https://doi.org/10.1007/s11269-021-02862-y}

The original version of this article unfortunately contained a mistake in Eqs. 1, 5 and 15. The original article has been corrected. The mistakes and corrections are described in the following list.

1. Equation 1,

$$
f_{\text {opt }}^{n}\left(S_{t}, Q_{t}\right)=\frac{\max }{R_{t}^{*}}\left[Z_{t}\left(S_{t}, Q_{t}, R_{t}\right)+\gamma f_{\text {opt }}^{-n}\left(S_{t+1}, Q_{t}\right)\right]
$$

should be

$$
f_{\text {opt }}^{n}\left(S_{t}, Q_{t}\right)=\max _{R_{t}^{*}}\left[Z_{t}\left(S_{t}, Q_{t}, R_{t}\right)+\gamma f_{\text {opt }}^{n-1}\left(S_{t+1}, Q_{t}\right)\right]
$$

2. Equation 5 ,

$p_{\theta}^{*}=\arg \frac{\min }{p_{\theta}} \mathrm{J}_{p_{\theta}}$ should be $p_{\theta}^{*}=\arg \min _{p_{\theta}} \mathrm{J}_{p_{\theta}}$

3. Equation 15 ,

$$
f_{\text {opt }}^{n}\left(S_{t}, Q_{t}\right)=\frac{\max }{R_{t}^{*}}\left[Z_{t}\left(S_{t}, Q_{t}, R_{t}\right)+\mathrm{E}_{Q_{t-1} \mid Q_{t}}\left(f_{\text {opt }}^{-n}\left(S_{t+1}, Q_{t+1}\right)\right)\right]
$$

should be

$$
f_{\text {opt }}^{n}\left(S_{t}, Q_{t}\right)=\max _{R_{t}^{*}}\left[Z_{t}\left(S_{t}, Q_{t}, R_{t}\right)+\underset{Q_{t+1} \mid Q_{t}}{E} f_{\text {opt }}^{n-1}\left(S_{t+1}, Q_{t+1}\right)\right]
$$

Publisher's Note Springer Nature remains neutral with regard to jurisdictional claims in published maps and institutional affiliations.

The original article can be found online at https://doi.org/10.1007/s11269-021-02862-y.

Young-Oh Kim

yokim05@snu.ac.kr

1 Department of Civil and Environmental Engineering, Seoul National University, Seoul 08826, South Korea 